\title{
ATTENUATION OF MAGNETOHYDRODYNAMIC OSCILLATIONS IN AN ALUMINUM REDUCTION CELL USING PERIODIC STRUCTURES
}

\author{
THAIS P. O. ANDERE* AND RODRIGO M. S. DE OLIVEIRA ${ }^{\dagger}$ \\ *Laboratory of Electromagnetics (LEMAG) \\ Universidade Federal do Pará \\ Caixa Postal 8619, R. Augusto Corrêa, 01 - Guamá, 66075-110, Belém - PA, Brasil \\ e-mail: thaispascoal@yahoo.com.br and rmso@ufpa.br \\ web page: http://www.lemag.ufpa.br/
}

Key words: Aluminum Reduction, MHD, Finite-difference time-domain (FDTD), Periodic Structures

\begin{abstract}
Magnetohydrodynamic instability (MHD) in an aluminum reduction cell is due to the interactions between the conductive liquid currents and the magnetic field generated by very high currents flowing through current feeding circuit buses. Such phenomenon creates oscillations in this fluid, compromising the efficiency of the process aluminum reduction. The reduction cells consist, in their usual configuration, of a container with flat walls that accommodates the liquid. In this work, a new geometry is proposed for the container based on periodic structures, with the aim of to mitigate such oscillations. The analysis of oscillations of fluid in both configurations is made with a software developed in this work to numerically simulate the process in two dimensions. The numerical formulation employed is based on the finite-difference time-domain method to solve the Navier-Stokes equations (N-S) explicitly through the Chorin projections method. The volume and surface of the fluid are mapped using the MAC method. The liquid is treated as incompressible and viscous, in addition to being electrically conductive. The accelerations caused by magnetic field and the electric currents are coupled to N-S by calculating the Lorentz Force. The results are analyzed by calculating differences between liquid heighs obtained at rest and by applying electric currents.
\end{abstract}

\section{INTRODUCTION}

The Magnetohydrodynamic effects caused by the interaction magnetic fields with an electrically conductive fluid are studied in many metallurgical processes to increase the control over the level of production and reduce costs [1]. The fact magnetic fields can influence the flow of electrically conductive fluids in various ways makes this subject much 
studied. Knowledge of the effects of Magnetohydrodynamics is a fundamental issue for the understanding of the aluminum production process, in which the behavior of liquid metals is strongly influenced by intense magnetic field and high currents that circulate through the fluid.

Aluminium is present into modern life, 1 billion tons os aluminium have been produced since 1886 and today much of it is still in use [2]. Much of the popularity of aluminum is due to its high electrical and thermal conductivities, in addition to its durability. Aluminum is used invarious applications such as: electrical installations, aircraft, automobile manufacturing, among many others.

Depending on the immense amount of energy used in the aluminum reduction process, small operational improvements scan produce very significant savings. For example, a reduction in voltage of $0.1 \mathrm{~V}$ in a single cell that operates with $100 \mathrm{kA}$ would save approximately $90.000 \mathrm{kWh}$ per year [3]. The process for the production of aluminum, actually, energy consumption of is ranged between $13-14 \mathrm{kWh} / \mathrm{kg}$ aluminium [4]. In a highly competitive industry like the aluminum industry, this is a significant quantity.

Aluminum is produced by electrolysis of aluminum oxide (alumina) in an aluminum reduction cell. At an inter-industrial environment, the Hall-Heroult process is used in the reduction cells.Large DC currents from 60 to $500 \mathrm{kA}$ flow through the cells. Strong magnetic fields ( $\mathrm{B} \approx 0.01 \mathrm{~T}$ ) are induced by currents that flow inside the cells and on the
busbars [5]. The process is maintained at temperatures from 950 ta $970^{\circ} \mathrm{C}$. These cells are
electrically connected in series through alunninum busbars. This circuit is called Potling.
Inside the cells, currents flow from the anode to the cathode. In an ideal situation, the
current inside the colls would be evenly distributed. This practically does not occur due
to the coupled various physical phenomena. There are signfficant differences between the to the coupled various physical phenomena. There are significant differences between the

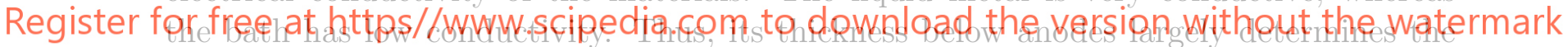

electrical and thermal behavior of the cell. The liquid bath floats on the aluminum liquid due to a small difference in density between the two liquids and it is responsible for the stability of the process. The electrical currents in the buses generate strong magnetic fields. The interaction of magnetic fields with the currents in the liquid metal generates electromagnetic forces that cause the two liquids move, thus deforming the interface between the bath and the metal.

A method of gaining deeper knowledge of the effects of operating parameters in the cell is the numerical representation, as practical tests are almost never viable, due to the need to interrupt the process of production. Numerical models can be used to a variety of purposes, such as determining depths of the metal and the bath, the concentration of alumina, operating temperature, assess corrosion of the anode and the structure of the cell walls. Another possibility is to study the electrical voltage to increase the operational current and, therefore, increase production, in order to reduce oscillations on the metal surface. These oscillations have frequencies determined by the size of the cavity and by electromagnetic characteristics. The typical period of these waves is 40 to 60 seconds. 
These waves are called instabilities. A large amount of energy is lost due to low bath conductivity (Joule effect). A way to restrict these losses is to maintain the distance between the anode and the interface $(A C D$ or anode-cathode distance) as small as possible. Unfortunately, it is very difficult [5].

There are two basic types of reduction cells: the Soderberg and the Prebake cell [6]. Soderberg cells have a large anode and Prebake smaller anodes. Inside each of these technologies, there are variations. An important variation is the amount of current flowing through a cell. Old cells have a low operating current $(75 \mathrm{kA})$ while current technology operates up to $500 \mathrm{kA}$. The size of the anodes, as well as their density, determines the operating current.

Haijun Sun [7] studied the stability of Hall-Heroult reduction cell using basic hydrodynamic equations the two-dimensional shallow water model, and applied the finite difference method to discretize them. JF Gerbeau [8] simulated the movement of fluids by using the Navier-Stokes equations coupled with Maxwell's equations and used the finite element technique.

In this work, two-dimensional Navier-Stokes equations numerical calculations are presented, which are applied to model in the magnetohydrodynamics of such liquids. The equations that describe the hydrodynamic behavior of the reduction cell, are solved nu-

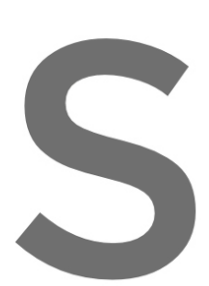
merically and discre Stokes Equations in method [9] was dev was used to study t. introduced in order
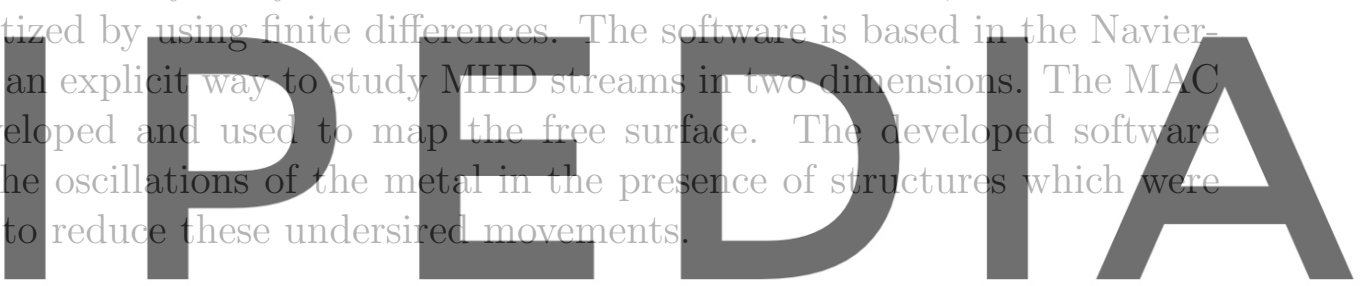

2 Mathematical and Numerical Modeling Register for free at https//www.scipedia.com to download the version without the watermark

Viscous and incompressible fluid flows are described by the following system of differential equations:

$$
\begin{gathered}
\frac{\partial \vec{u}}{\partial t}+(\vec{u} \cdot \nabla) \vec{u}=-\frac{1}{\rho} \nabla p+\nu \nabla^{2} \vec{u}+\vec{a}, \\
\nabla \cdot \vec{u}=0 .
\end{gathered}
$$

The equation (1) is known as the equation of conservation of momentum and (2) is called the continuity equation. Such a system is called the Navier-Stokes equations, in which $t$ is time $(\mathrm{s}), \vec{u}=(u, v, w)$ is the flow velocity vector $(\mathrm{m} / \mathrm{s}), p$ is the scalar pressure field $(\mathrm{Pa}), \rho$ and $\nu$ are respectively the density $\left(\mathrm{kg} / \mathrm{m}^{3}\right)$ and the kinematic viscosity $\left(\mathrm{m}^{2} / \mathrm{s}\right)$ of the fluid and $\vec{a}$ represents external accelerations, such as the electromagnetic acceleration $\vec{a}_{m}$ and the gravitational acceleration $\vec{a}_{g}\left(\mathrm{~m} / \mathrm{s}^{2}\right)$. It is worth noting that in this paper, the term $\vec{a}$ is used as a movement source. 
For the present problem, illustrated by Figure 1, the walls are considered to be impermeable and non-slip. Therefore, the boundary conditions

$$
\begin{aligned}
& u_{n}=0 \\
& u_{t}=0,
\end{aligned}
$$

were employed for the velocity, where the subscripts $n$ and $t$ indicate, respectively, the normal and tangential components of $\vec{u}$ to the considered solid surfaces. The boundary conditions (3) ensures impermeability and (4) ensures that the obstacle is not slippery.

To numerically solve the system formed by (1) and (2), the MAC [9] (marker and cell) methodology associated with finite difference discretization and the scheme presented in [10] are employed. The time derivative of $\vec{u}$ is approximated by finite differences by

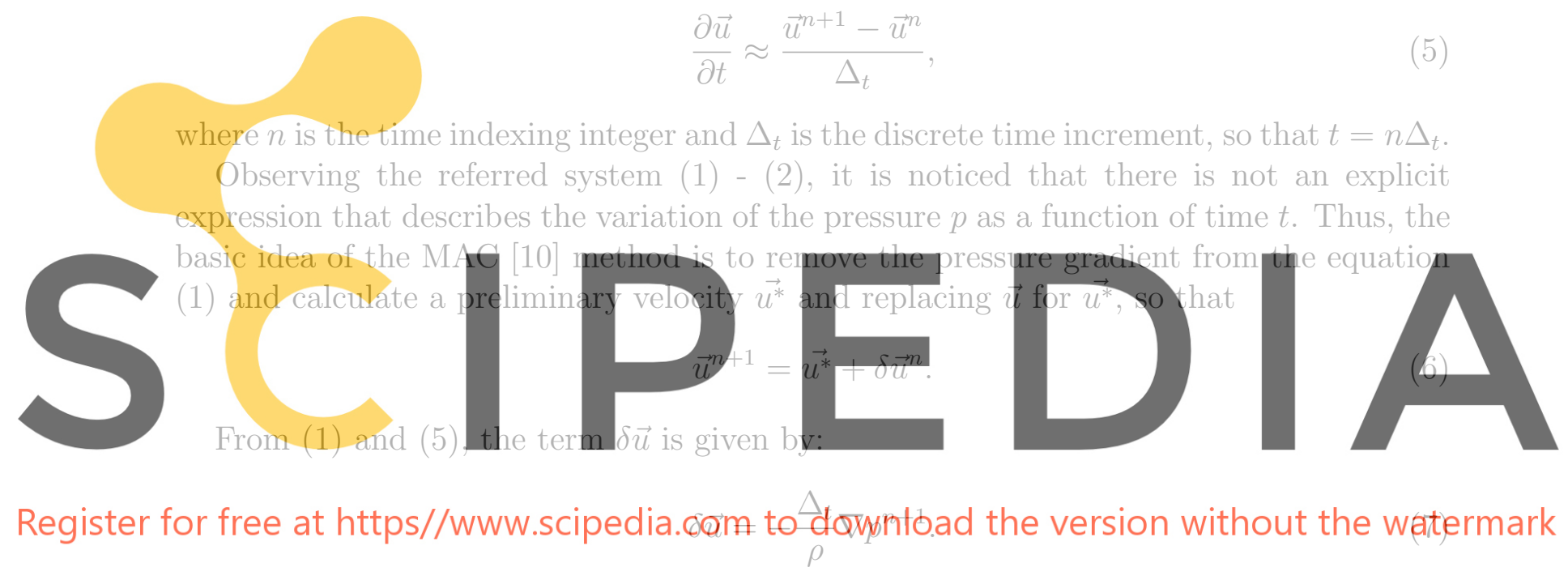

The expression for $p$ at time $n+1$ can be found by substituting (7) in (6) and then applying (2), which provides

$$
\nabla^{2} p^{n+1}=\frac{\rho}{\Delta_{t}} \nabla \cdot \overrightarrow{u^{*}}
$$

In this way, there is an explicit update scheme for the velocity and an implicit pressure update scheme.

The equation (8) is subjected to the boundary condition $\partial p / \partial \vec{n}=0$ on the solid walls present in the domain, which avoids fluid accelerations in normal directions to these obstacles. Equation (8) is solved, in this work, by successive over-relaxation method [11].

Thus, the basic algorithm of the fluid dynamic model can be synthesized by the iterative process described below.

\section{Algorithm I}

- calculate $\vec{a}$ (see algorithm II); 
- calculate $\overrightarrow{u^{*}}$ using (1) by ignoring the term $-\frac{1}{\rho} \nabla p$;

- solve equation (8);

- update the velocity field $\vec{u}$ using (7) and (6);

- return to the first step as long as there is no convergence of the obtained solution.

\subsection{The Electromagnetic Model}

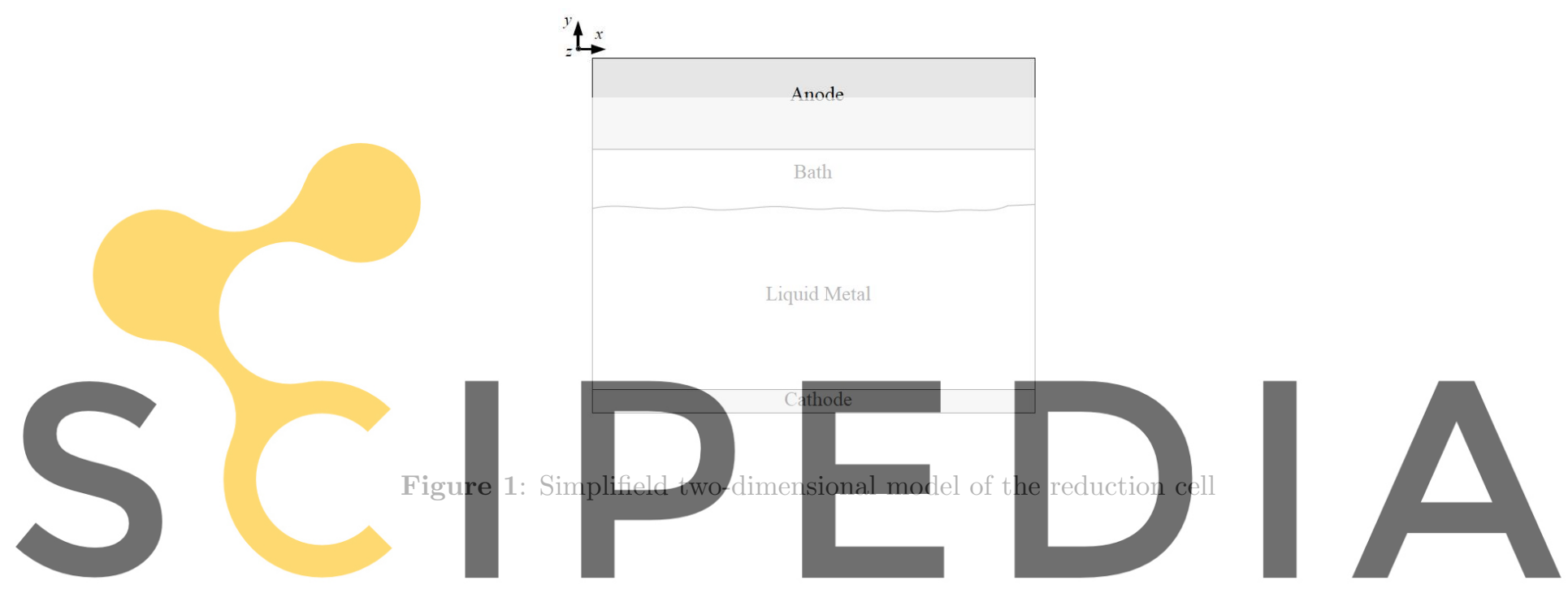

Maxwell's equations, which govern electromagnetic phenomena [12], can be written for Register for freatat.hłtps//www.scipedia.com to download the version without the watermark

$$
\nabla \times \vec{e}=0
$$

and

$$
\nabla \times \vec{b}=\mu_{m} \vec{j}
$$

where $\vec{e}$ is the electric field intensity vector $(\mathrm{V} / \mathrm{m}), \vec{b}$ is the flux density vector (Tesla), $\mu_{m}$ is the magnetic permeability (Henry/m) and $\vec{j}$ is the electric current density vector $\left(\mathrm{A} / \mathrm{m}^{2}\right)$.

From the equations (9) and (10), it is observed mathematically that

$$
\vec{e}=-\nabla \phi
$$

and

$$
\nabla \cdot \vec{j}=0
$$

where $\phi$ is, by definition, the electric scalar potential (V). 
The electric current density $\vec{j}$ is given by Ohm's law

$$
\vec{j}=\sigma(\vec{e}+\vec{u} \times \vec{b})
$$

where $\sigma$ is the electrical conductivity of the liquid $(\mathrm{S} / \mathrm{m})$.

If the equation (11) is used in (13), it is obtained, using (12)

$$
\nabla^{2} \phi=\nabla \cdot(\vec{u} \times \vec{b})
$$

For the problem illustrated by Fig. 1, (14) is subjected to the boundary conditions $\phi=5 \mathrm{~V}$ at the anode, $\phi=0 \mathrm{~V}$ at cathode and $\partial \phi / \partial \vec{n}=0$ in solid outlines. This equation is then solved by the over-relaxation method [11].

Thus, after calculating the current density distribution $\vec{j}$, it is possible to determine the Lorentz acceleration $\vec{a}_{m}$, which is given by

$$
\vec{a}_{m}=\frac{\vec{j} \times \vec{b}}{\rho} .
$$

Thus, the basic algorithm for updating the electromagnetic acceleration that acts in the conducting liquid can besynthesized by
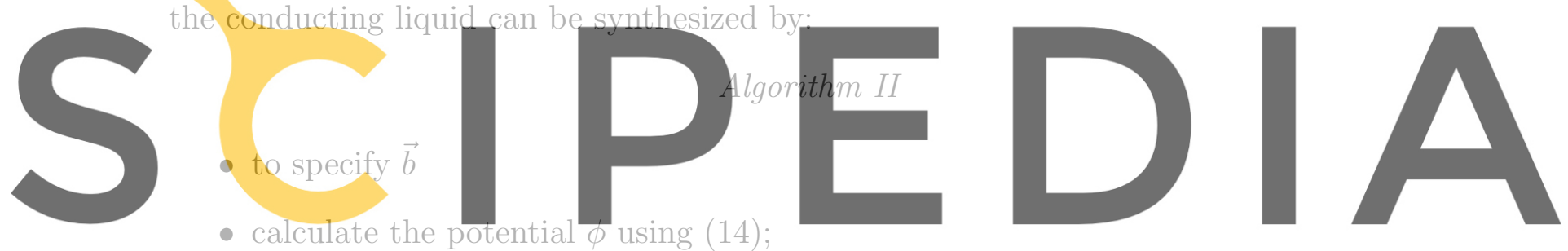

Register for freenatehttesp/(Aw)ww.scipedia.com to download the version without the watermark

- update $\vec{j}$ using (13);

- compute $\vec{a}_{m}$ given by (15).

\subsection{Treatment of equations}

\subsubsection{Dimensionless CFD Equations}

Defining the parameters $\vec{X}=\vec{x} / x_{0}, \dot{\nabla}=x_{0} \nabla, P=p /\left(\rho u_{0}^{2}\right), \vec{U}=\vec{u} / u_{0}, T=t u_{0} / x_{0}$, $\vec{A}_{g}=\vec{a}_{g} x_{0} / u_{0}^{2}, \vec{B}=\vec{b} / b_{0}, \Phi=\phi /\left(u_{0} b_{0} x_{0}\right), \vec{E}=-\dot{\nabla} \Phi, \vec{J}=\vec{E}+\vec{U} \times \vec{B}$ and $\vec{A}_{m}=\vec{J} \times \vec{B}$, it is possible to show, by substitution, that (1) and (2) in their dimensionless forms, are given by

$$
\frac{\partial \vec{U}}{\partial T}+(\vec{U} \cdot \dot{\nabla}) \vec{U}=-\dot{\nabla} P+\frac{1}{R e} \dot{\nabla}^{2} \vec{U}+\vec{A}_{g}+N \vec{A}_{m}
$$

and

$$
\dot{\nabla} \cdot \vec{U}=0
$$


where $R e=x_{0} u_{0} / \nu$ is known as the Reynolds number [13], $N=\sigma b_{0}^{2} x_{0} /\left(\rho u_{0}\right)$ is called number of Stuart [14] and $\overrightarrow{A_{g}}$ and $\overrightarrow{A_{m}}$ represent normalized magnetic and gravitational accelerations, respectively. The subscript 0 indicates the reference values.

By following the same procedure, (7) and (8) can be written adimensionally as

$$
\delta \vec{U}=-\Delta_{T} \dot{\nabla} P^{n+1}
$$

and

$$
\dot{\nabla}^{2} P^{n+1}=\frac{1}{\Delta_{T}} \dot{\nabla} \cdot \vec{U}^{*}
$$

respectively. Thus, the flow is characterized by only two parameters [14], Re and $N$.

\subsubsection{Spatial Discretization}

The computational cell used to spatially represent the fields velocity and pressure follows [10] and it is illustrated by Figure 2. The fields, $U$ and $V$ are, respectively, the $X$ and $Y$ components of $\vec{U}$ in cell $(i, j)$. Figure 2 also shows the positioning of the electrical potential $\Phi$, of the components of the vector $\vec{J}$ and the $Z$-component of the $\vec{B}$ vector. This positioning can be obtained through the analysis of the equations (11) - (15).
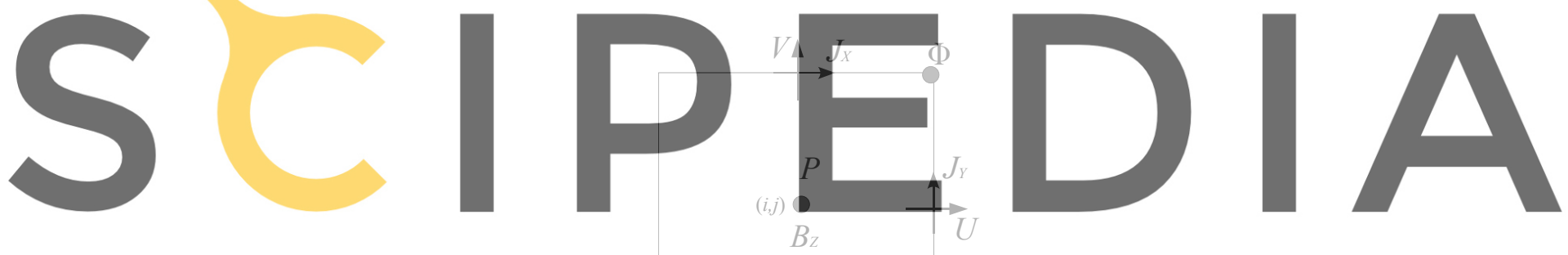

Register for free at https//www.scipedia.com to download the version without the watermark

Figure 2: The two-dimensional finite difference cell

Thus, without loss in generality, we have for the two-dimensional case, for the $X$ component of $\vec{U}$ from (16), removing the pressure gradient and using (5), the finite difference equation

$$
U_{(i, j)}^{*}=U_{(i, j)}^{n}+\Delta_{T}\left(\frac{1}{R e} \dot{\nabla}^{2} U-\frac{\partial U V}{\partial Y}-\frac{\partial U^{2}}{\partial X}+G_{X(i, j)}^{n}+N A_{X(i, j)}^{n}\right),
$$

in which

$$
\begin{array}{r}
\frac{\partial U^{2}}{\partial X} \approx\left[\left(U_{(i, j)}^{n}+U_{(i+1, j)}^{n}\right) \cdot\left(U_{(i, j)}^{n}+U_{(i+1, j)}^{n}\right)+\gamma\left|U_{(i, j)}^{n}+U_{(i+1, j)}^{n}\right| \cdot\left(U_{(i, j)}^{n}-U_{(i+1, j)}^{n}\right)\right. \\
\left.-\left(U_{(i-1, j)}^{n}+U_{(i, j)}^{n}\right) \cdot\left(U_{(i-1, j)}^{n}+U_{(i, j)}^{n}\right)-\gamma\left|U_{(i-1, j)}^{n}+U_{(i, j)}^{n}\right| \cdot\left(U_{(i-1, j)}^{n}-U_{(i, j)}^{n}\right)\right] /\left(4 \Delta_{X}\right),
\end{array}
$$




$$
\begin{array}{r}
\frac{\partial U V}{\partial Y} \approx\left[\left(V_{(i, j)}^{n}+V_{(i+1, j)}^{n}\right) \cdot\left(U_{(i, j)}^{n}+U_{(i, j+1)}^{n}\right)+\gamma\left|V_{(i, j)}^{n}+V_{(i+1, j)}^{n}\right| \cdot\left(U_{(i, j)}^{n}-U_{(i, j+1)}^{n}\right)\right. \\
\left.-\left(V_{(i, j-1)}^{n}+V_{(i+1, j-1)}^{n}\right) \cdot\left(U_{(i, j-1)}^{n}+U_{(i, j)}^{n}\right)-\gamma\left|V_{(i, j-1)}^{n}+V_{(i+1, j-1)}^{n}\right| \cdot\left(U_{(i, j-1)}^{n}-U_{(i, j)}^{n}\right)\right] /\left(4 \Delta_{Y}\right)
\end{array}
$$

and

$$
\dot{\nabla}^{2} U \approx\left[U_{(i+1, j)}^{n}-2 U_{(i, j)}^{n}+U_{(i-1, j)}^{n}\right] / \Delta_{X}^{2}+\left(U_{(i, j+1)}^{n}-2 U_{(i, j)}^{n}+U_{(i, j-1)}^{n}\right) / \Delta_{Y}^{2},
$$

where the term $\gamma$ is called the upwind [15] coefficient and $V$ represents $v$ in dimensionless form.

From (19), we have

$$
P_{(i, j)}=(1-\omega) P_{(i, j)}+-\beta\left[\left(P_{(i+1, j)}+P_{(i-1, j)}\right) / \Delta_{X}^{2}+\left(P_{(i, j+1)}+P_{(i, j-1)}\right) / \Delta_{Y}^{2}-\psi_{(i, j)}\right],
$$

where $\omega$ is the relaxation coefficient [11], $\beta=-\omega\left[2\left(\Delta_{X}^{-2}+\Delta_{Y}^{-2}\right)\right]^{-1}$ and

$$
\psi_{(i, j)}=\frac{1}{\Delta_{T}}\left[\left(U_{(i, j)}^{*}-U_{(i-1, j)}^{*}\right) / \Delta_{X}+\left(V_{(i, j)}^{*}-V_{(i, j-1)}^{*}\right) / \Delta_{Y}\right]
$$

The equations (20) and (20) are computed several times until the convergence is found for all values $P_{(i, j)}$ in the computational grid. The final values found are used to calculate
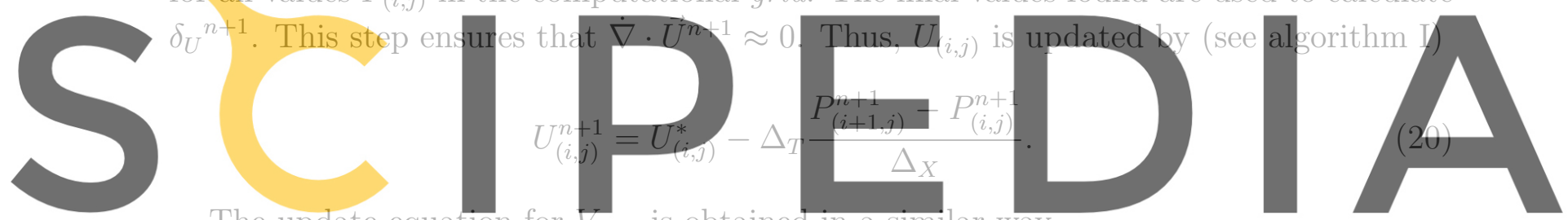

The update equation for $V_{(i, j)}$ is obtained in a similar way.

Register for free at https//Wwww.scipedia.com to download the version without the watermark

To guarantee numerical stability, the scheme must satisfy the condition

$$
\Delta_{T} \leq \frac{R e}{2\left(\Delta_{X}^{-2}+\Delta_{Y}^{-2}\right)+N R e}
$$

as it is discussed in details in [16].

\subsubsection{Free Surface}

To map the surface oscillations of the conductive liquid and determining the fluid domain, massless particles were used [9], which move according to the components of the nearest $\vec{U}$ velocity field.

The equation

$$
\frac{\partial \vec{X}}{\partial T}=\vec{U}
$$


governs the movement of particles. In $(22), \vec{U}$ is the result of the 2 D interpolation of $\vec{U}$ [17], calculated for each particle. In finite differences, the equation (22) is approximated by

$$
X^{n+1}=X^{n}+\Delta_{T} \bar{U}^{n}
$$

and

$$
Y^{n+1}=Y^{n}+\Delta_{T} \bar{V}^{n}
$$

where $X$ and $Y$ are normalized $x$ and $y$ coordinates.

At each time step, the cells in the domain need to be reclassified, for the correct application of boundary conditions. The basic classification of cells is done as follows:

- filled (with fluid),

- empty,

- superficial,

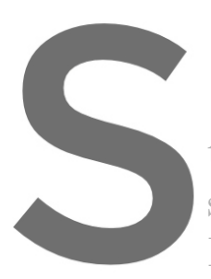

- solid contour

If a cell $(i, j)$ contains one or more particl
perficial. If the current cell $(i, j)$ does not
superficial cells that contain only one f
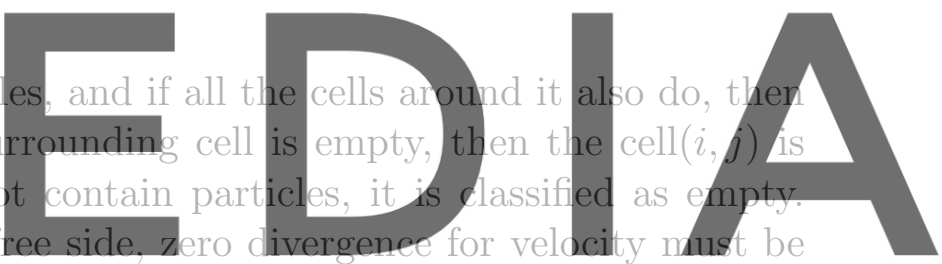

guaranteed using (17) for the component of that cell face. For two free faces, each spatial

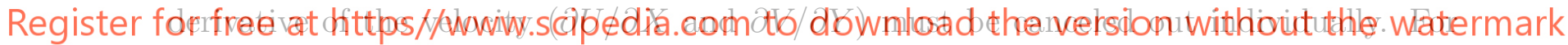
the case of three free faces, the velocity component on the side opposite the fluid receives the value of the fluid component and the other two feel the effects caused by the external acceleration from its previous values. The pressure receives the value of atmospheric pressure for all surface cells. Further details on these boundary conditions are presented in $[18]$.

\section{Results}

\subsection{Validation Hydrodynamic Simulations}

In this work, some results for incompressible flows with and without free surfaces are shown to validate the use of the methodology. Two problems widely used for testing numerical methods for hydrodynamics are that of the controlled cavity shown in Figure 3(a) and that of a drop of fluid falling into a container containing the same fluid shown in Figure 4(a). The results obtained were compared with classic works. In both cases, the results show excellent agreement with those shown in the works by Krzeminski et al. [19], for the controlled cavity problem, and that of Foster et al. [17], for the free surface 
problem. The parameters used in the simulations are the same as those in reference articles used for validation.

Figure 3(a) represents the simulation of a fluid in a controlled cavity for $R e=100$. In this simulation, a uniform-structured mesh was used containing $n x=20$ cells for $x$-direction and $n y=20$ cells for $y$-direction with spacing of $\Delta x=\Delta y=0.05$.

Figure 4(a) represents a simulation of a drop of fluid immersing in a container that contains the same fluid for $R e=1500, n x=50, n y=40$ and $\Delta x=\Delta y=0.02$.
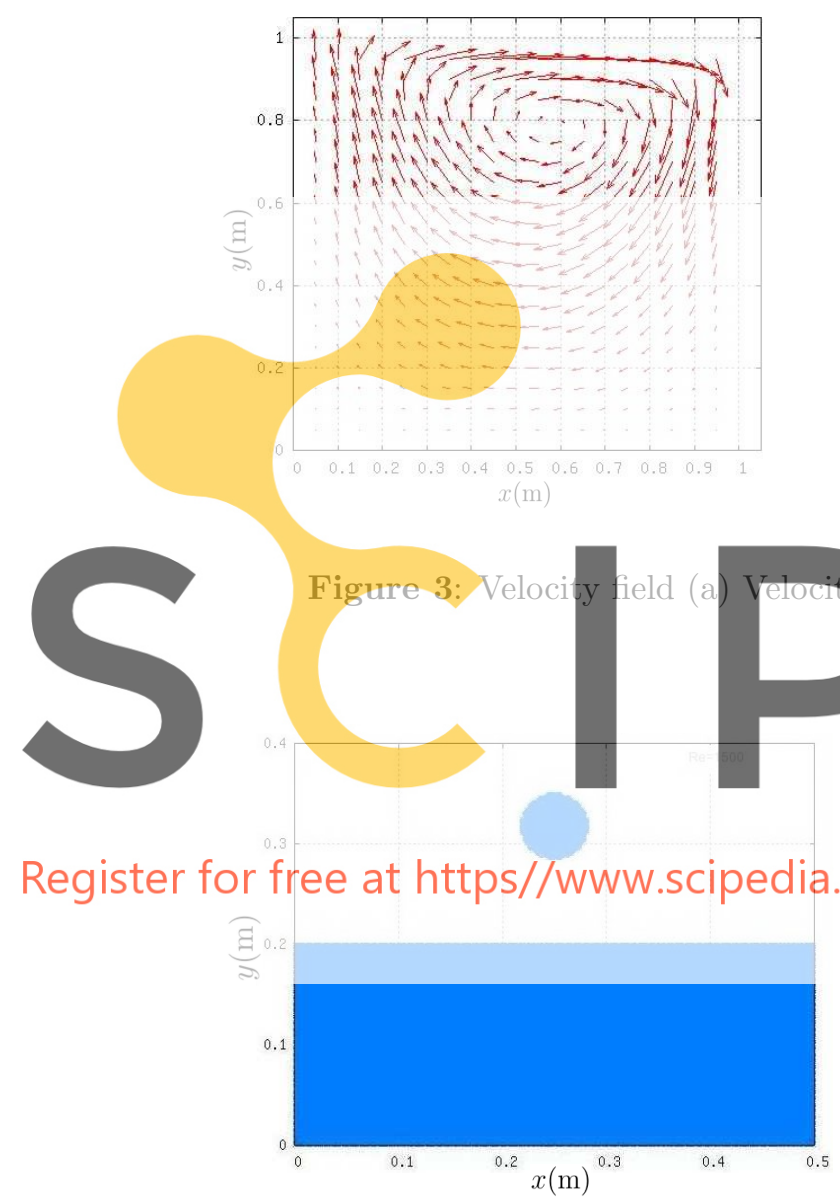
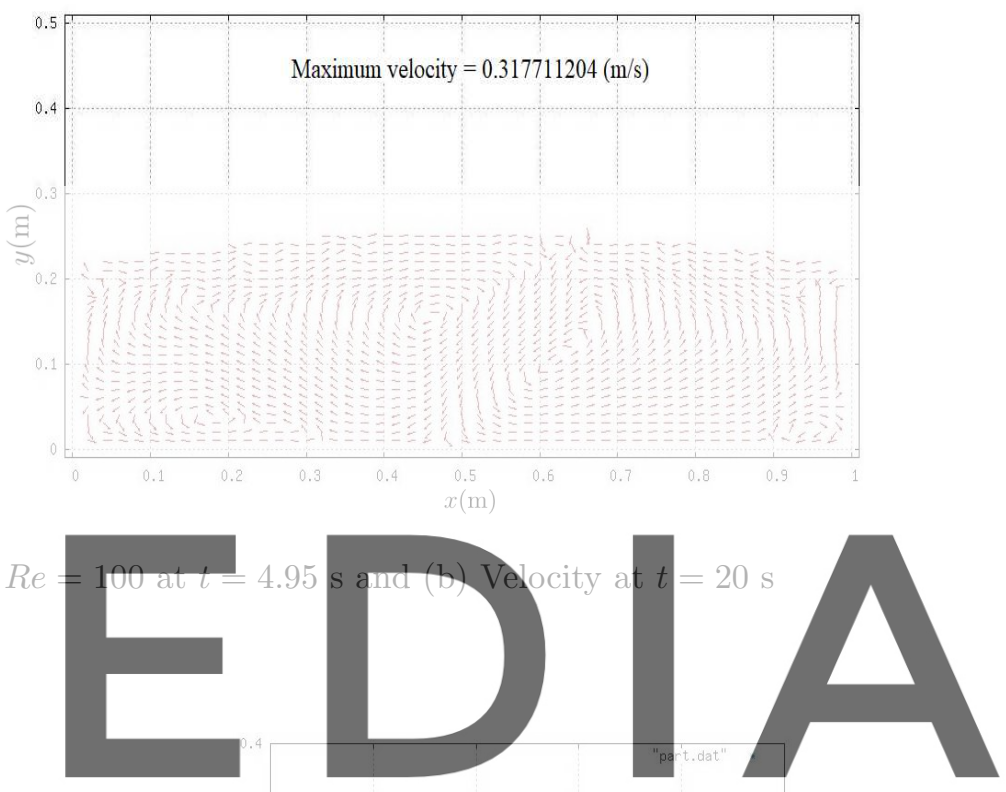

Register for free at https//www.scipedia.com to download the version without the watermark

Figure 4: Drop in free fall (a) Drop in free fall for $R e=1500$ and $t=0 \mathrm{~s}$ and (b) Drop in free fall for $R e=1500$ and $t=0.4 \mathrm{~s}$.

\subsection{Magnetohydrodynamic Simulations}

This section presents simulations made with the objective of proving the efficiency of periodic structures applied to the side walls of a cell. The simulations showed excellent results, demonstrating that the maximum height that the metal reached, over time, with walls with periodic structures is smaller than in cells with flat walls. In the simulations, 
the following parameters were considered: $n x=100, n y=50, \Delta x=\Delta y=0.01 \mathrm{~m}$, $R e=1.923 \times 10^{6}$ and $N=891.3$.

Figure 3(b) shows the velocity profile in the cell for the situation shown in Figure 5(a). The cell is one meter wide and the metal net starts at a height of $0.2 \mathrm{~m}$. $B z(X)$, magnetic field, used is a linear function of $X$, so that, in the center $(x=0,5 \mathrm{~m}), B z=0$.
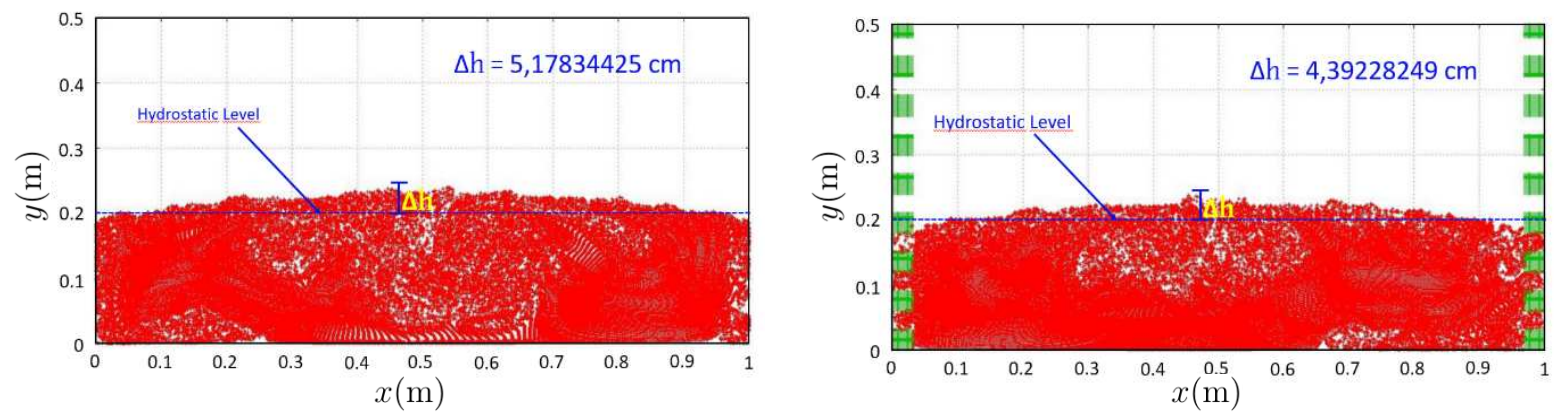

Figure 5: Fluid behavior (a) Cells without periodic walls (maximum height of $5.178 \mathrm{~cm}$ ) and (b) Cells with periodic walls (maximum height of $4.392 \mathrm{~cm}$ ).

\section{Conclusions}

The results obtained in this work show that the developed numerical model can be used to describe the behavior of aluminum in a reduction cell. Using the numerical this model, simulations were carried out confirming that cells with periodic walls are more effective, since the height of the liquid has been reduced, which implies greater cell stability and, as a consequence, higher aluminium reduction efficiency can be obtained.

\section{REFERENCES}

[1] P. A. Davidson, Magnetohydrodynamics in materials processing, Annu. Rev. Fluid Mech, Vol. 31, pp. 273-300, (1999).

[2] The Aluminium Association, https://www.thealuminiumstory.com/, (accessed May 11, 2021).

[3] P. Biedler, Modeling of an Aluminum Reduction Cell for the Development of a State Estimator, College of Engineering and Mineral Resources, (2003).

[4] M. Dupuis, How to limit the heat loss of anode stubs and cathode collector bars in order to reduce cell energy consumption, TMS light Metals, pp. 521-531, (2019).

[5] H. Sun, O. Zikanova, D. P. Ziegler, Non-linear two-dimensional model of melt flows and interface instability in aluminum reduction cells, Fluid Dynamics Research, Vol. 35, pp. 255-274, (2004). 
[6] Galevskiy, G. V., V. V. Rudneva, and S. G. Galevskiy. Production of prebaked anodes for aluminum electrolysis cells: dominant trends, technology, quality, IOP Conference Series: Materials Science and Engineering. Vol. 866. No. 1, (2020).

[7] H. Sun, O. Zikanova, B. A. Finlayson, The Influence of base flow on aluminum reduction cell's stability, Light Metals, (2005).

[8] J. F. Gerbeaua and T. Leli Vreb and C. Le Brisb, Simulations of MHD flows with moving interfaces, Journal of Computational Physics, Vol. 184, pp. 163-191, (2003).

[9] F. H. , Harlow and J. E., Welsh, Numerical calculation of time-dependent viscous incompressible flow of fluid with free surface, Physics of Fluids, Vol. 8, pp. 21822189, (1965).

[10] A. J. Chorin, Numerical solution of the Navier-Stokes equations, Mathematics of Computation, Vol. 22, pp. 745-762, (1968)

[11] M. N. O. Sadiku, Numerical Techniques in Electromagnetics, CRC Press, second edition, (2000).

[12] J. D., Jackson, Classical Electrodynamics, Wiley, New York, (1962).

[13] P. A. Davidson, An introduction to Magnetohydrodynamics, Cambridge University Press, (2001).

[14] D. P. O. Zikanov and A. Thess, Direct numerical simulation as a tool for understanding MHD liquid metal turbulence, Applied Mathematical Modelling, Vol. 28, pp. 1-13, (2004).

[15] W. Hackbusch, Elliptic differential equations, theory and numerical treatment, Spring-Verlag, (1992)

[16] L. Leboucher, Monotone Scheme and Boundary Conditions for Finite Volume Simulation of Magnetohydrohynamic Internal Flow at High hartmann Number, Journal of Computational Physics, Vol. 150, pp. 181-198, (1999)

[17] N. Foster and D. Metaxas, Realistic Animation of Liquids, Graphical Models and Image Processing, Vol. 58, pp. 471-483, (1996).

[18] M. Tomé and S. McKee, GENSMAC: A computational marker and cell method for free surface flows in general domains", Journal of Computational Physics, Vol. 110, pp. 171-186, (1994).

[19] S. K. Krzeminski and M. Smialek, and M. Wlodarczyk, Finite Element Approximation of Biharmonic Mathematical Model for MHD Flow Using qsi - An Approach. IEEE Transactions on Magnetics, Vol. 36, (2000). 